\section{Constructing cDNA Libraries with Fewer Clones that Contain Long poly(dA) Tails}

BioTechniques 31:38-42 (July 2001)

cDNA libraries are usually constructed by reverse transcription of mRNA using an oilgo(dT) primer (5). Some of the cDNAs thus synthesized may contain long poly (dA) tails because the oligo(dT) primer can anneal to any region of the poly(A) tail of an mRNA transcript. The cDNAs that contain long poly(dA) tails are difficult to sequence from the $3^{\prime}$ ends with universal vector primers $(3,4)$. We found that 3 ' sequencing of cDNA clones containing poly (dA) tails longer than $40 \mathrm{nu}-$ cleotides [designated as long poly(dA) cDNA clones] usually yielded unreadable signal for the region downstream from the poly(dA) tail (Figure 1). Although a long poly(dA) cDNA may be sequenced by using an anchored oligo(dT) primer $(3,4)$, such an approach is inappropriate for large-scale sequencing of randomly picked cDNA clones for the purpose of generating expressed sequence tags (ESTs) $(1,2)$ because some of the randomly picked cDNA clones may not contain poly(dA) tails. Therefore, alternative methods are necessary to reduce the number of long poly(dA) cDNA clones when constructing cDNA libraries for large-scale sequencing. We have found two methods useful for constructing cDNA libraries with fewer long poly(dA) clones during the preparation of cDNA libraries for a large-scale porcine EST project (http://ascswine. rnet.missouri.edu).

The first method involves using a 10-fold mass excess of oligo(dT) primer in the reverse transcription reaction. An excess of oligo(dT) primer will theoretically saturate the poly(A) tails of the mRNA templates so that only the primer annealed closest to the $5^{\prime}$ end of the poly(A) tail will be extended by the reverse transcriptase (6). However, the amounts of primer needed to saturate the poly(A) tail were unknown. Assuming an mRNA population with an average size of $1.2 \mathrm{~kb}$, a 100-200-nucleotide poly(A) tail, and a primer of 60 nucleotides, of which 20 nucleotides are oligo(dT), a given amount of this oligo(dT) primer will theoretically provide at least a 2-fold molar excess relative to the poly(A) tails of an equal amount of the mRNA. Based on this calculation, we initially used a 1:1 mass ratio of oligo(dT) primer to mRNA (usually $1 \mu \mathrm{g}$ primer and $1 \mu \mathrm{g}$ mRNA) in the reverse transcription reaction. The sequence of a representative oligo(dT) primer used in our study was 5'-GACTAGTTCTAGATCGCGAGCGGCCGCACGTTTTTTTTTTTTTTTTTTTTTTTT-3', in which the first 27 nucleotides were used to form restriction sites and the following six nucleotides were used to form a library tag. We performed the reverse transcription reaction and the remaining steps of library construction by using the SUPERSCRIPT TM Plasmid System for cDNA Synthesis and Plasmid Cloning according to the manufacturer's instructions (Life Technologies, Rockville, MD, USA). DNA sequencing revealed that $23.2 \%$ of the cDNA clones with poly(dA) tails were long poly(dA) clones when libraries were synthesized using a 1:1 mass ratio of oligo(dT) primer to mRNA (Table 1). This result suggested that nearly onequarter of the poly(A) tails in the mRNA sample were not saturated by the primer, even though a 1:1 ratio of oligo(dT) primer to mRNA theoretically provided at least a 2 -fold molar excess needed to anneal to all of the poly(A) tails. We then increased the primer to mRNA mass ratio to $10: 1$ in the reverse transcription reaction. The cDNA library made using a 10:1 mass ratio of primer to mRNA contained $11.1 \%$ long poly(dA) clones (Table 1). This result indicates that at least a 10fold excess of oligo(dT) primer needs to be used in the reverse transcription reaction to saturate $90 \%$ of the poly(A) tails. In our 3' EST project, the cDNA libraries that contain $10 \%$ or less long poly(dA) clones were acceptable for large-scale sequencing (http:// ascswine.rnet.missouri.edu).

The second method that we have found useful to construct cDNA li-

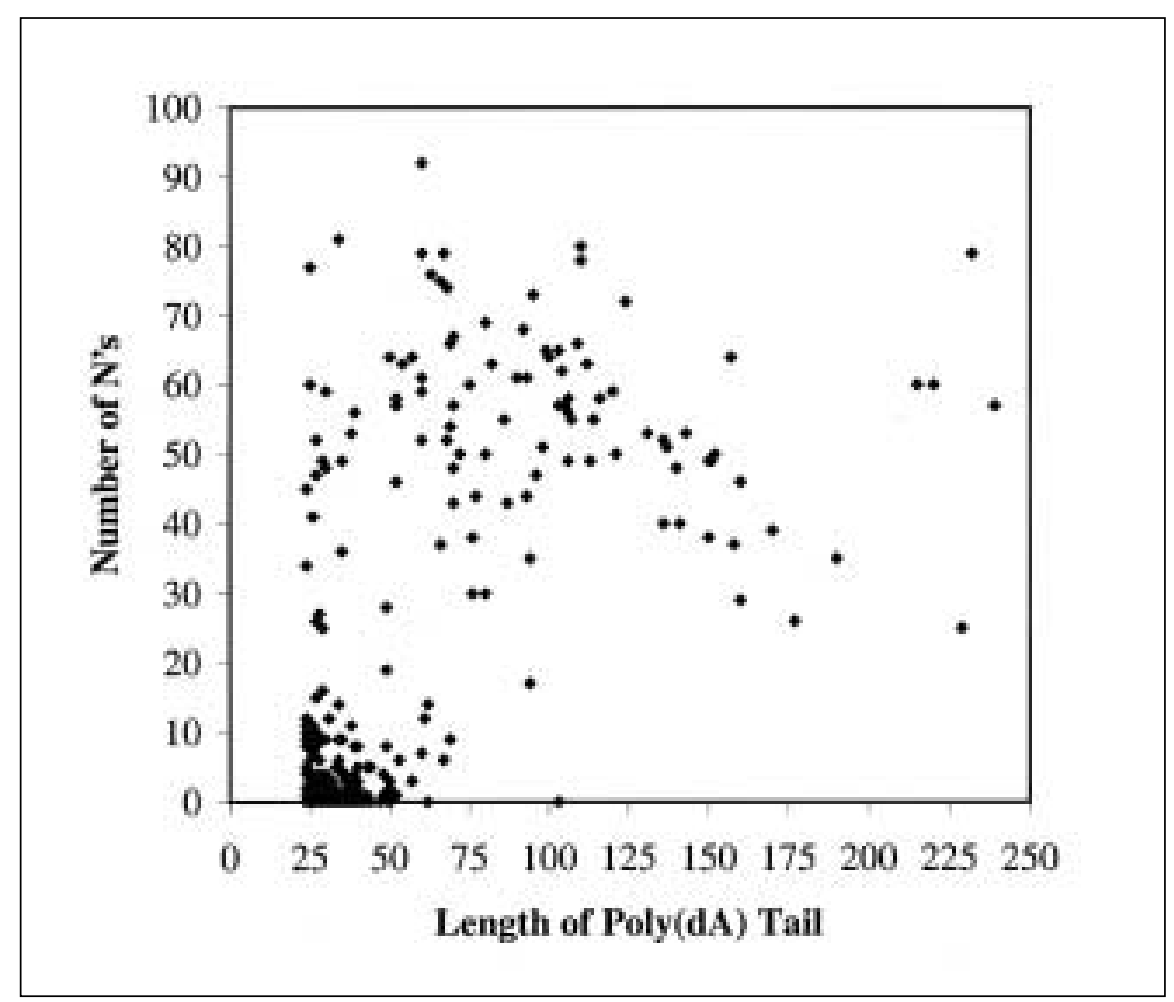

Figure 1. cDNAs with long poly(dA) tails are difficult to sequence from their $\mathbf{3}^{\prime}$ ends. cDNAs (405) with poly (dA) tails between 24 and 239 nucleotides were sequenced using an M13 forward primer. The number of Ns represents the number of unreadable nucleotides within 200 nucleotides downstream from the poly(dT) stretch. 


\section{Benchmarks}

braries with fewer long poly $(\mathrm{dA})$ cDNA clones involves using an anchored oligo(dT) primer in the reverse transcription reaction and performing the reverse transcription reaction at a relatively high temperature of $50^{\circ} \mathrm{C}$. The sequence of a representative anchored oligo(dT) primer used in our study was 5'-GACTAGTTCTAGATCGCGAGCGGCCGCACGTTATTTTTTTTTTTTTTTTTTTTTTTTVN-3', where $\mathrm{V}=\mathrm{A}, \mathrm{C}$, or $\mathrm{G}$ and $\mathrm{N}=\mathrm{A}, \mathrm{C}, \mathrm{G}$, or $\mathrm{T}$. Compared to the conventional oligo(dT) primer, the anchored oligo(dT) primer contained two additional nucleotides at the $3^{\prime}$ end of the oligo(dT) stretch. These two additional nucleotides were expected to anchor the primer at the $5^{\prime}$ end of the poly(A) tail of the mRNA. We initially performed the reverse transcription reaction with this anchored primer at $37^{\circ} \mathrm{C}$. Sequencing of randomly picked clones revealed that the cDNA libraries made using the anchored oligo(dT) primers at $37^{\circ} \mathrm{C}$ contained approximately $5 \%$ fewer long poly $(\mathrm{dA})$ clones than the libraries

Table 1. Percentages of Long poly(dA) Clones in cDNA Libraries Constructed by Using Two Different Ratios of oligo(dT) Primer to mRNA

\begin{tabular}{|ccc|}
\hline & \multicolumn{2}{c|}{ Primer:mRNA } \\
\cline { 2 - 3 } Library Attempt & $\mathbf{1 : 1}$ & $10: 1$ \\
\hline 1 & $28.3(58 / 205)^{\mathrm{a}}$ & $10.6(22 / 207)$ \\
2 & $15.5(33 / 213)$ & $4.9(7 / 144)$ \\
3 & $23.7(49 / 207)$ & $11.2(25 / 224)$ \\
4 & & $13.5(19 / 141)$ \\
5 & $23.2 \pm 2.7$ & $11.1 \pm 1.9$ \\
Mean \pm SE & & \\
a28.3\% or 58 out of 205 cDNAs with poly(dA) tails contained long poly(dA) tails. \\
bMean \pm SE of percentages of long poly(dA) cDNA clones from libraries of differ- \\
ent attempts. Means for 10:1 and 1:1 differed at $P<0.05$ ( $t$ test). \\
\hline
\end{tabular}

Table 2. Percentages of Long poly(dA) Clones in cDNA Libraries Constructed by Using an Anchored oligo(dT) Primer [oligo(dT)VN] at Two Different Incubation Temperatures for the Reverse Transcription Reaction

\begin{tabular}{|c|c|c|}
\hline \multirow[b]{2}{*}{ Library Attempt } & \multicolumn{2}{|c|}{ Temperature } \\
\hline & $37^{\circ} \mathrm{C}$ & $50^{\circ} \mathrm{C}$ \\
\hline 1 & $19.7(46 / 233)$ & $8.6(16 / 187)$ \\
\hline 2 & $17.6(47 / 267)$ & $10.2(25 / 245)$ \\
\hline 3 & & $8.7(22 / 252)$ \\
\hline 4 & & $6.3(12 / 192)$ \\
\hline 5 & & $5.9(14 / 239)$ \\
\hline Mean \pm SEa & $18.6 \pm 1.5$ & $8.0 \pm 1.8$ \\
\hline
\end{tabular}

made using conventional oligo(dT) primers (Tables 1 and 2). However, approximately $18.6 \%$ of the poly $(\mathrm{dA})$ cDNA clones in the libraries constructed using the anchored oligo(dT) primer still contained long poly $(\mathrm{dA})$ tails (Table 2). This percentage was still too high for large-scale DNA sequencing. By examining the sequenced poly $(\mathrm{dA})$ stretches, we found that some anchored oligo(dT) primers, especially those with $3^{\prime}$ nucleotides CT and AT, could anneal to the downstream region of the $\operatorname{poly}(\mathrm{A})$ tails when the incubation temperature was $37^{\circ} \mathrm{C}$ and that these mismatched primers were successfully extended by the reverse transcriptase (data not shown). To reduce nonspecific annealing of the primer to the mRNA template, we performed the reverse transcription reaction at $50^{\circ} \mathrm{C}$, the up- per temperature limit for the enzymatic activity of the SUPERSCRIPT reverse transcriptase. The cDNA sequencing confirmed that more anchored oligo(dT) primers annealed to the $5^{\prime}$ ends of the poly $(\mathrm{A})$ tails at $50^{\circ} \mathrm{C}$. Consequently, only $8 \%$ of the poly(dA) cDNA clones in these libraries had long poly(dA) tails (Table 2). This percentage was significantly lower than the percentage of long poly (dA) clones in the cDNA libraries made using the same anchored oligo(dT) primer with an annealing temperature of $37^{\circ} \mathrm{C}(P<$ 0.05) (Table 2).

In summary, we found that two methods can be used to construct cDNA libraries with a minimum number of long poly(dA) cDNA clones. The first method involves using at least a 10-fold mass excess of oligo(dT) primer relative to the 


\section{Benchmarks}

mRNA in the reverse transcription reaction. The second method involves using an anchored oligo(dT) primer in the reverse transcription and performing the reverse transcription at a relatively high temperature of $50^{\circ} \mathrm{C}$. The cDNA libraries made using either method contained approximately $10 \%$ long poly(dA) clones compared to approximately $25 \%$ long poly(A) clones contained in the cDNA libraries made using conventional conditions (1:1 mass ratio of primer to mRNA and the temperature for the reverse transcription reaction at $37^{\circ} \mathrm{C}$ ). The cDNAs synthesized under the modified conditions did not appear to differ in yield, size distribution, or frequency of polyadenylation signals from the cDNAs made under conventional conditions (data not shown). The cDNA libraries made with these modified conditions will be more cost effective when used for large-scale $3^{\prime}$ sequencing because the percentages of cDNAs yielding poor sequences are reduced.

\section{REFERENCES}

1.Adams, M.D., J.M. Kelley, J.D. Gocayne, M. Dubnick, M.H. Polymeropoulos, H. Xiao et al. 1991. Complementary DNA sequencing: expressed sequence tags and human genome project. Science 252:1651-1656.

2.Hillier, L.D., G. Lennon, M. Becker, M.F. Bonaldo, B. Chiapelli, S. Chissoe et al. 1996. Generation and analysis of $280,000 \mathrm{hu}-$ man expressed sequence tags. Genome Res. 6:807-828.

3.Khan, A.S., A.S. Wilcox, J.A. Hopkins, and J.M. Sikela. 1991. Efficient double stranded sequencing of cDNA clones containing long poly(A) tails using anchored poly(dT) primers. Nucleic Acids Res. 19:1715.

4.Liao, J. and Z. Gong. 1997. Sequencing of 3' cDNA clones using anchored oligo(dT) primers. BioTechniques 23:368-370.

5.Sambrook, J., E.F. Fritsch, and T. Maniatis. 1989. Molecular Cloning. A Laboratory Manual, 2nd ed. CSJ Laboratory Press, Cold Spring Harbor, NY.

6.Soares, M.B. and M.F. Bonaldo. 1998. Construction and screening of normalized cDNA libraries, p. 49-157. In B. Birren, E.D. Green, S. Klapholz, R. Myers, J. Roskams (Eds.), Genome Analysis: A Laboratory Manual. CSH Laboratory Press, Cold Spring Harbor, NY.

The authors thank Dr. M.B. Soares at University of Iowa for helpful discussions. The authors also thank Bronwen Blaue and Brandi McCormack for technical assistance. This work was supported by a grant from the Monsanto Company. Address correspondence to Dr. Honglin Jiang, 164 Animal Science Research Center, University of Missouri, Columbia, MO 65211, USA. e-mail: jiangh@missouri.edu

Received 23 January 2001; accepted 17 April 2001.
H. Jiang, N.J. Bivens, J.E. Ries, K.M. Whitworth, J.A. Green, L.J. Forrester, G.K. Springer, B.A. Didion', N. Mathialagan', R.S. Prather, and M.C. Lucy
University of Missouri
Columbia, $M O$
${ }^{1}$ Monsanto Company
St. Louis, MO, USA

\section{SYBR ${ }^{\circledR}$ Green I Used to Evaluate the Nuclei Num- ber of Fungal Mycelia}

BioTechniques 31:42-46 (July 2001)

The species concept of Rhizoctonia solani Kühn specifies that isolates must have the following traits: multinucleated cells in young vegetative hyphae, dolipore septa, branching near the distal septum of cells in young vegetative hyphae, constriction at the base of the branching, formation of a septum in the branch near the point of origin, and brown pigmentation (8). Fungi that possess the above-mentioned characteristics, with the exception that the majority of the young vegetative hyphal cells are bi- or trinucleated, are designated Ceratobasidium spp. (9).

To observe the number of nuclei within these two fungal species, several techniques have been used to grow and stain cultures. Cultures have been grown on water agar, removed and stained with $0.5 \%$ aniline blue in lactophenol, and examined with a standard light microscope (2) or grown on water agar-coated slides and stained with the following: a mixture of $50 \mu \mathrm{g} / \mathrm{mL}$ acridine orange and $0.5 \mathrm{ng} / \mathrm{mL}$ ethidium bromide (1), $0.0025 \%$ acridine orange in veronal acetate buffer (11) or DAPI (6), and examined via a fluorescence microscope. In all cases, water agar is present and can interfere with the staining and visualization of the sample. Furthermore, in the case of DAPI, the samples are fixed in 3\% formaldehyde before staining, and the water agar absorbs the stain, thus requiring additional washing of the samples (6). The amount of washing is especially important because all of the listed fluorescent stains are mutagenic and pose environmental concerns for their treatment and safe disposal. Another technique utilizes a cellophane strip, which supports the fungus as it grows over the surface of the media (7). The cellophane strip is removed from the surface and placed on a microscope slide before staining. Cellophane can interfere with the staining and can cause background fluorescence. To overcome these problems, we developed an improved method of cul- 